\title{
Effects of Convective Heating on Movement and Vertical Coupling of Tropical Cyclones: A Numerical Study*
}

\author{
LIGUANG WU AND BIN WANG \\ Department of Meteorology, School of Ocean and Earth Science and Technology, University of Hawaii at Manoa, Honolulu, Hawaii
}

(Manuscript received 25 February 2000, in final form 15 June 2001)

\begin{abstract}
The influence of convective heating on movement and vertical coupling of tropical cyclones (TCs) is investigated using a hurricane model with different environmental flows. The authors identify two processes by which convective heating may affect TC motion. One is the advection of symmetric potential vorticity (PV) by heatinginduced asymmetric flow. The other is the direct generation of a positive PV tendency by asymmetric heating, which acts to shift a TC to the region of maximum downward gradient of asymmetric heating. A steering level exists that is located at the level where the direct influence of asymmetric heating vanishes, normally in the lower troposphere. At that level, a TC moves with the asymmetric flow averaged within a radius of $200 \mathrm{~km}$, because the influence of asymmetric flows on TC motion is weighted by the horizontal PV gradient that is primarily confined within the TC core. Although the vertical shear in the asymmetric flow (including environmental and heating-induced flows) could tilt the vortex, the influence of asymmetric heating tends to offset the vertical tilt caused by the vertical shear through a fast adjustment between the asymmetric wind and diabatic heating. Therefore, diabatic heating enhances the vertical coupling.
\end{abstract}

\section{Introduction}

Diabatic heating is a fundamental physical process determining tropical cyclone (TC) evolution. Recently, efforts have been made through numerical experiments to understand its roles in TC movement and in maintaining the TC vertical structure (the vertical coupling) (e.g., Shapiro 1992; Flatau et al. 1994; Wang and Holland 1996b,c; Dengler and Reeder 1997). It has been found that the diabatic heating associated with the convection within a TC can significantly affect TC motion and vertical coupling in comparison with the corresponding adiabatic experiments, although the convection parameterization schemes used in the previous studies differ from one model to another. In the beta drift problem, for example, Flatau et al. (1994) and Wang and Holland (1996b) showed that in the Northern Hemisphere the diabatic heating leads to a more northward TC track than without heating. Furthermore, the vertical tilt of the baroclinic TC circulation is significantly re-

\footnotetext{
* This is the School of Ocean and Earth Science and Technology Publication 5830 and International Pacific Research Center Publication 97. The International Pacific Research Center is sponsored in part by the Frontier Research System for Global Change.
}

Corresponding author address: Dr. Bin Wang, Department of Meteorology and International Pacific Research Center, University of Hawaii at Manoa, 2525 Correa Road, Honolulu, HI 96822. E-mail: bwang@soest.hawaii.edu duced compared to that in the corresponding adiabatic experiments.

The mechanisms responsible for the influence of diabatic heating on TC motion and vertical coupling have been primarily explained in terms of the asymmetric flow induced by diabatic heating, a concept originally established from barotropic TC motion (Fiorino and Elsberry 1989). In the presence of diabatic heating, a complex potential vorticity $(\mathrm{PV})$ pattern occurs at the upper levels (Shapiro 1992; Wang and Holland 1996b; Shapiro and Franklin 1995; Wu and Kurihara 1996). The negative PV anomaly at the upper levels can induce a downward penetration flow, affecting TC motion (Wu and Emanuel 1993). Wang and Holland (1996b) argued that since the center of the upper-level anticyclone is displaced horizontally from the TC center, downward penetration of the circulation by these anticyclonic PV anomalies slows down the westward motion of diabatic vortices. The meandering of cyclone tracks has also been attributed to the oscillation of axially asymmetric convection (e.g., Neumann and Boyd 1962; Willoughby et al. 1984; Holland and Lander 1993). In a shallow water model, Willoughby (1992) found that the vortex center was deflected due to the asymmetric convectioninduced divergent wind associated with the specified mass sink and source. Using a five-level model, Wang and Holland (1996b) confirmed that the asymmetric divergent flow within the vortex core can deflect the vortex center toward the region of maximum convection. 
On the other hand, evidence has shown that the steering of the resulting asymmetric flow is not enough to account for the influence of diabatic heating on TC motion. For instance, using a PV tendency diagnostic analysis of adiabatic baroclinic TC motion, Wu and Wang (2001) showed that TC motion can substantially deviate from the mean asymmetric flow averaged over the TC core region. The TC motion is also determined by nonsteering processes: horizontal advection of the asymmetric PV component associated with the symmetric flow and the vertical advection of the symmetric PV component associated with the asymmetric vertical motion. Furthermore, both observational (Wu and Emanuel 1995a,b) and numerical (Wang and Holland 1996b; Bender 1997) studies indicate that the asymmetric steering flow over the TC core region varies considerably with height, yet the TC maintains coherent vertical structure. This enhancement of vertical coupling cannot be interpreted with the induced asymmetric flows as suggested by Wang and Holland (1996b). Therefore, the processes responsible for the enhanced vertical coupling need to be better understood.

In this paper, we further examine the roles of diabatic heating in TC motion and vertical coupling with the PV tendency diagnostic approach. Specifically, the following questions are addressed. 1) How does the diabatic heating affect TC motion? 2) How can a TC maintain its coherent vertical structure in the presence of significant vertical shears of the asymmetric flow? Since with different convection schemes one might have different cyclone tracks for the same experiments performed in this paper, the focus of this paper is placed on the physical mechanism, not on the specific behaviors of TC motion that are affected by different environmental flows.

In section 2, three idealized numerical experiments and the corresponding TC tracks are described, in which the TC motion is respectively affected by a zonal uniform flow, a vertical environmental shear, and the effect of vertical differential beta drift. The individual contributions of diabatic heating and other processes to TC motion in these experiments are assessed in section 3. Section 4 discusses the mechanism by which diabatic heating affects TC motion. Section 5 discusses the mechanism for the vertical coupling. Major findings are summarized in section 6 .

\section{Experimental design and TC tracks}

A hurricane model, designed by Wang (1998), is used in this study. The cumulus convection is parameterized with Kuo's (1974) scheme. The details of the model and its capability to simulate the motion and evolution of baroclinic TCs in the presence of diabatic heating has been documented by Wu (1999). The model domain consists of $201 \times 201$ grid points with a uniform horizontal resolution of $25 \mathrm{~km}$ and 16 vertical layers.

Idealized numerical experiments are run with differ- ent environmental flows on an $f$ plane or a beta plane. Due to the similar conclusions, three experiments are addressed here. The axially asymmetric circulation is critical to TC motion. In those typical experiments, different mechanisms are considered to initiate asymmetric circulation in the presence of diabatic heating. All three experiments begin with the same initially symmetric baroclinic vortex and the SST remains horizontally uniform and fixed $\left(27.5^{\circ} \mathrm{C}\right)$ throughout the integration. The first diabatic experiment (E1) is designed on an $f$ plane with a uniform easterly environmental flow of $-4 \mathrm{~m}$ $\mathrm{s}^{-1}$. According to Tuleya and Kurihara (1984), superposition of a uniform environmental wind on TC circulation in the planetary boundary layer can provide flux asymmetries that would enhance the diabatic heating in the region with relatively high winds. As a result, asymmetric circulation can be induced. The second experiment (E2) is designed to focus on the influence of vertical environmental shears on an $f$ plane. In E2, the environmental flow decreases with height from $-4 \mathrm{~m}$ $\mathrm{s}^{-1}$ at the surface to $0 \mathrm{~m} \mathrm{~s}^{-1}$ at the top of the model domain. According to Raymond (1992) and Frank and Ritchie (1999), such a vertical environmental shear can generate a mesoscale asymmetric circulation by interacting with the TC's symmetric heating source. Even without diabatic heating, the vertical tilt of the initially symmetric vortex due to the shear of the environmental flows over the TC center may induce a three-dimensional asymmetric circulation (Jones 1995; Wang and Holland 1996b). The vertical shear can significantly affect TC motion (Shapiro 1992; Wu and Emanuel 1993; Flatau et al. 1994; Wang and Holland 1996c; Dengler and Reed 1997). The third experiment (E3) is run on a beta plane with a resting environment, focusing on the influence of diabatic heating on the beta drift, which has been discussed to some extent in previous studies (e.g., Wang and Li 1992; Shapiro 1992; Wang and Holland 1996a,b). Due to the heating-induced upper-level anticyclonic circulation and the decrease of the cyclonic vortex strength with height, the vertical differential beta effect can produce strong vertical shears in the asymmetric flow over the TC center, thus indicating interaction between the asymmetric flow and diabatic heating.

The 72-h vortex centers defined by the positions of maximum PV at the lowest model level in all the three experiments are shown in Fig. 1. The center in E1 moves $1197 \mathrm{~km}$ to the west and $152 \mathrm{~km}$ to the north. The mean speed is $4.6 \mathrm{~m} \mathrm{~s}^{-1}$ westward and $0.6 \mathrm{~m} \mathrm{~s}^{-1}$ northward, which is obviously faster than the environmental easterly flow of $4 \mathrm{~m} \mathrm{~s}^{-1}$. It is interesting that the TC in a uniform easterly flow (E1) propagates northward. Despite the presence of the vertical shear in the environmental easterly flow, the vortex in E2 takes a track similar to that in E1 but at a slower speed. In $\mathrm{E} 2$ the center moves $855 \mathrm{~km}$ to the west and 113 $\mathrm{km}$ to the north. Such a northward propagation (to the left of the environmental vertical shear) has been pre- 


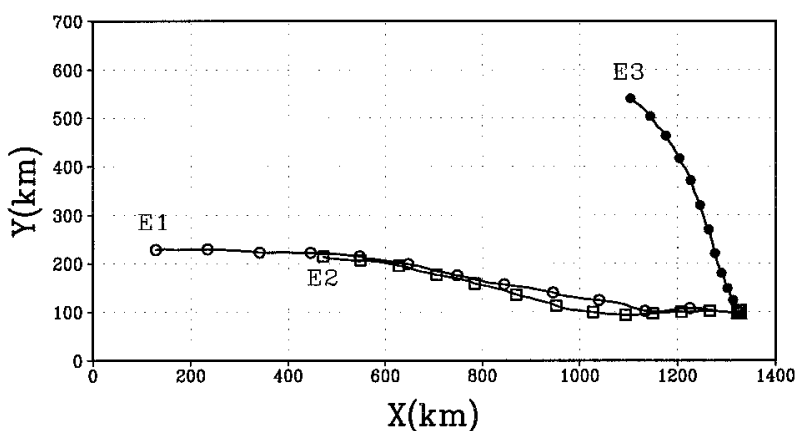

FIG. 1. 72-h tracks of the surface centers with 6-h positions indicated by circles for E1, squares for E2, and closed circles for E3. The initial position is indicated by a large closed square.

viously found by $\mathrm{Wu}$ and Emanuel (1993), Flatau et al. (1994), and Wang and Holland (1996c) in models with various degrees of complexity. In E3, the center drifts $222 \mathrm{~km}$ to the west and $440 \mathrm{~km}$ to the north. Compared to the experiment without heating, the heating slows down the westward beta drift, whereas it accelerates the northward beta drift, in agreement with Wang and Holland (1996b).

It is found that significant vertical tilt only occurs at the levels above $365 \mathrm{hPa}$ (level 6). For example, Fig. 2 shows east-west cross sections of PV after a 48-h integration in these experiments. Despite the westerly vertical shear in E2 and the differential beta forcing in $\mathrm{E} 3$, the positive PV anomalies of the TCs are vertically aligned very well.

\section{Contributions of diabatic heating and other processes to TC motion}

Although the TCs in these three diabatic experiments maintain their coherent vertical structures, their centers are not precisely vertically aligned. Vertical tilts at the upper levels are evident (Fig. 2). In this study, we view a three-dimensional TC as a stack of two-dimensional vortices in the vertical. The advantages are as follows. First, since the center positioning is carried out at each vertical level, it can avoid unrealistic asymmetric circulation arising from the variation of the TC center with height (Willoughby 1988). Second, it facilitates investigation of the physical mechanism behind the vertical coupling, because the horizontal positions of the TC center are allowed to vary with height. If there is a mechanism by which the vortices at different levels navigate different centers, we can identify the mechanism using the PV tendency diagnostic approach (Wu and Wang 2000). Third, since we consider the vortex movement at various vertical levels, we can determine the contribution of a certain physical process to the vortex movement at a single level and its contribution to the three-dimensional TC motion, respectively.

Treated as a localized positive PV anomaly relative to the environmental $\mathrm{PV}$, the $\mathrm{TC}$ motion is taken as the

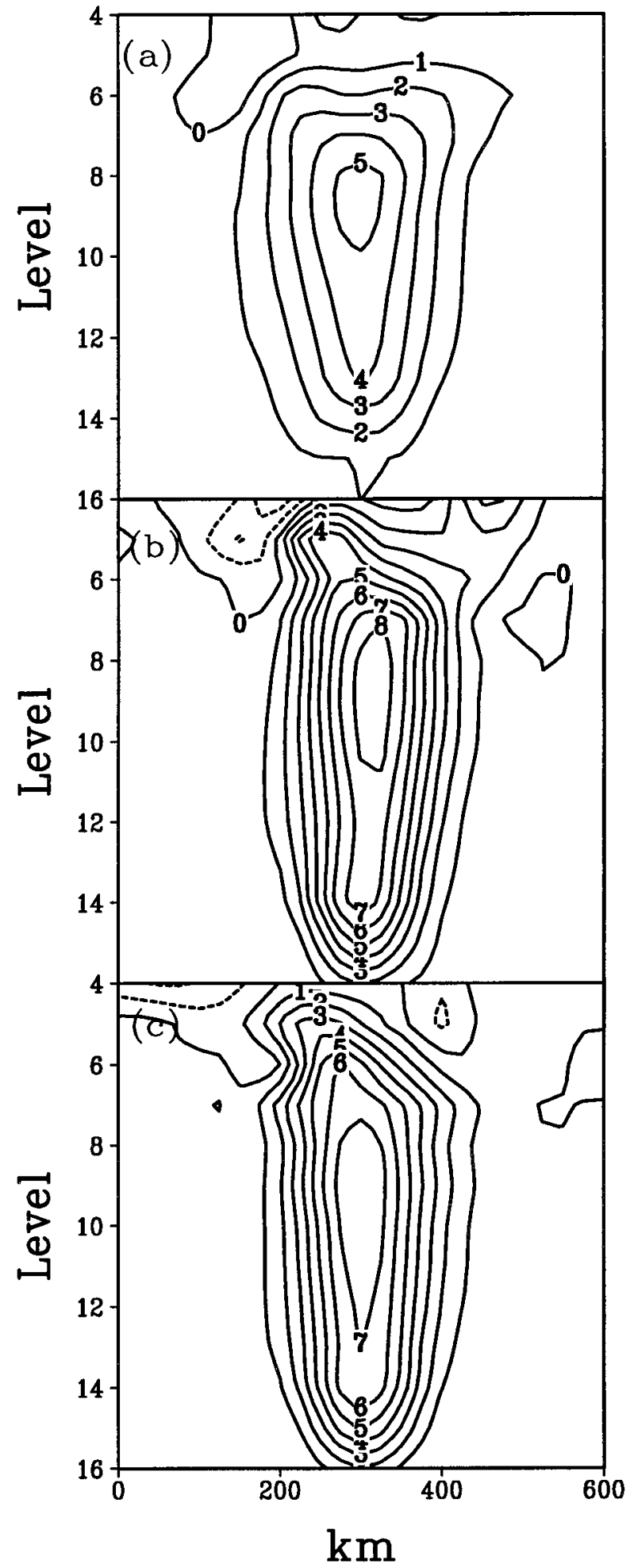

FIG. 2. The east-west cross sections of PV at $48 \mathrm{~h}$ in (a) E1, (b) $\mathrm{E} 2$, and (c) E3. The contour intervals are 1.0 PVU $\left(10^{-6} \mathrm{~m}^{2} \mathrm{~s}^{-1} \mathrm{~K}\right.$ $\left.\mathrm{kg}^{-1}\right)$. 
movement or propagation of the positive PV anomaly. In this section, we first look at the contributions of various physical processes to the two-dimensional vortex motion at each level (hereafter the vortex motion), and then the three-dimensional TC motion (hereafter the TC motion). The latter is defined as the vertical mean motion of vortices at the vertical levels. The mean motion speed is calculated based on the vertical extent of the positive PV anomaly shown in Fig. 2.

\section{a. Contributions to the vortex motion}

$\mathrm{Wu}$ and Wang (2000) demonstrated that the vortices at the levels where the symmetric circulation is dominant move to the region with the maximum wavenumber-one component of the PV tendency, $(\partial P / \partial t)_{1}$. Therefore, the contributions of individual physical processes to the vortex motion are equivalent to their contributions to the wavenumber-one component of the PV tendency, which can be calculated from the following $\mathrm{PV}$ tendency equation

$$
\begin{aligned}
\left(\frac{\partial P}{\partial t}\right)_{1}=\Lambda_{1} & -\mathbf{V} \cdot \boldsymbol{\nabla} P-\dot{\sigma} \frac{\partial P}{\partial \sigma} \\
& \left.-\frac{g}{p_{s}} \nabla_{3} \cdot\left(-\frac{Q}{C_{p} \pi} \mathbf{q}+\boldsymbol{\nabla} \theta \times \mathbf{F}\right)\right],
\end{aligned}
$$

where $P$ represents $\mathrm{PV} ; \mathbf{V}$ and $\dot{\sigma}$ are the horizontal and the vertical velocities in $\sigma$ coordinates; $\mathbf{q}$ is the threedimensional absolute vorticity vector; $\Lambda_{1}$ denotes an operator to obtain the wavenumber-one component; $\pi$ is the Exner function; $p_{\mathrm{s}}$ is the surface pressure; $\boldsymbol{\nabla}_{3}$ is the three-dimensional gradient operator; and $Q$ and $\mathbf{F}$ denote diabatic heating rate and friction, respectively.

As shown in Eq. (1), there are four processes that can contribute to the wavenumber-one component of the PV tendency: horizontal PV advection, vertical PV advection, diabatic heating, and friction. The details of the calculations of their contributions are described by $\mathrm{Wu}$ and Wang (2000). The domain used to estimate the contributions of various physical processes is a square area of $400 \mathrm{~km} \times 400 \mathrm{~km}$, since the PV anomaly associated with a TC is primarily confined within such an area (Fig. 2). Here we examine the contributions of these processes to the vortex motion at levels $9(580 \mathrm{hPa})$, $11(730 \mathrm{hPa})$, and $13(850 \mathrm{hPa})$. Since the influence of friction on the vortex motion is very small, Fig. 3 only shows the contributions of horizontal PV advection (HA), vertical PV advection (VA), and diabatic heating (HE) at $48 \mathrm{~h}$. As suggested by Willoughby (1992) and Wang and Holland (1996b), the asymmetric diabatic heating can induce asymmetric flows. Note that the influence associated with the heating-induced asymmetric flows is implicitly included in the contribution of horizontal PV advection. For this reason, the diabatic heating term $\mathrm{HE}$ will be referred to as the direct effect of the heating on motion.
In E1, as shown in Fig. 3, the vortices move westward with a moderate northward component. Due to the easterly environmental flow, the contribution of HA plays the most important role in the vortex motion. Note that here HA is not simply advection by the uniform environmental flow. It also includes the flow induced by diabatic heating. However, the contribution of $\mathrm{HE}$ is also important to vortex motion, especially at lower levels (11 and 13). The diabatic heating leads the vortices toward the southwest of the vortex centers. In E2, the diabatic heating leads to a southwestward motion at levels 9 and 11, but a northward motion at level 13. In this case, the meridional component induced by diabatic heating acts against the influence of the horizontal advection. In E3, due to absence of the environmental flow, the horizontal PV advection and the diabatic heating play nearly the same role in the vortex motion at levels 9 and 11. At level 13, however, the vortex motion is dominated by the contribution of the horizontal PV advection. Overall, as shown in Fig. 3, the vortex motion is primarily determined by the contributions of horizontal PV advection and diabatic heating. Their contributions can vary considerably with height.

As pointed out by Wu and Wang (2001), the influence of horizontal advection can be further partitioned into the advection of the wavenumber-one PV component by the symmetric flow and the advection of the symmetric PV component by the wavenumber-one flow component. The latter can be referred to as the steering by the asymmetric flow, which can be approximately expressed as the area-averaged mean asymmetric flow in the same domain over which the contribution of horizontal PV advection is estimated. In the adiabatic experiments, Wu and Wang (2001) showed that the total contribution of horizontal advection is significantly different from the mean asymmetric flow $\left(\mathbf{V}_{m 1}\right)$. However, in the presence of diabatic heating, the areal mean asymmetric flow can, in general, represent well the total contribution of horizontal PV advection to the vortex motion (figures not shown).

As shown in Fig. 3, in addition to the implicit effect of the heating-induced asymmetric flow on horizontal PV advection, the diabatic heating can directly play an important role in the vortex motion. Figure 4 shows the fields of asymmetric diabatic heating rate and the corresponding vertical gradient of asymmetric heating rate indicated by the difference of asymmetric diabatic heating rate between two adjacent levels. Rather than shifting to the region with maximum heating rate, the direct effect of diabatic heating leads vortices to the regions with the maximum vertical gradient of asymmetric diabatic heating. Therefore, the direct influence of heating on TC motion is primarily determined by the vertical gradient of asymmetric diabatic heating.

\section{b. Contributions to the TC motion}

Figure 5 shows the vertically averaged TC speeds calculated from the vortex center positions (thick solid 


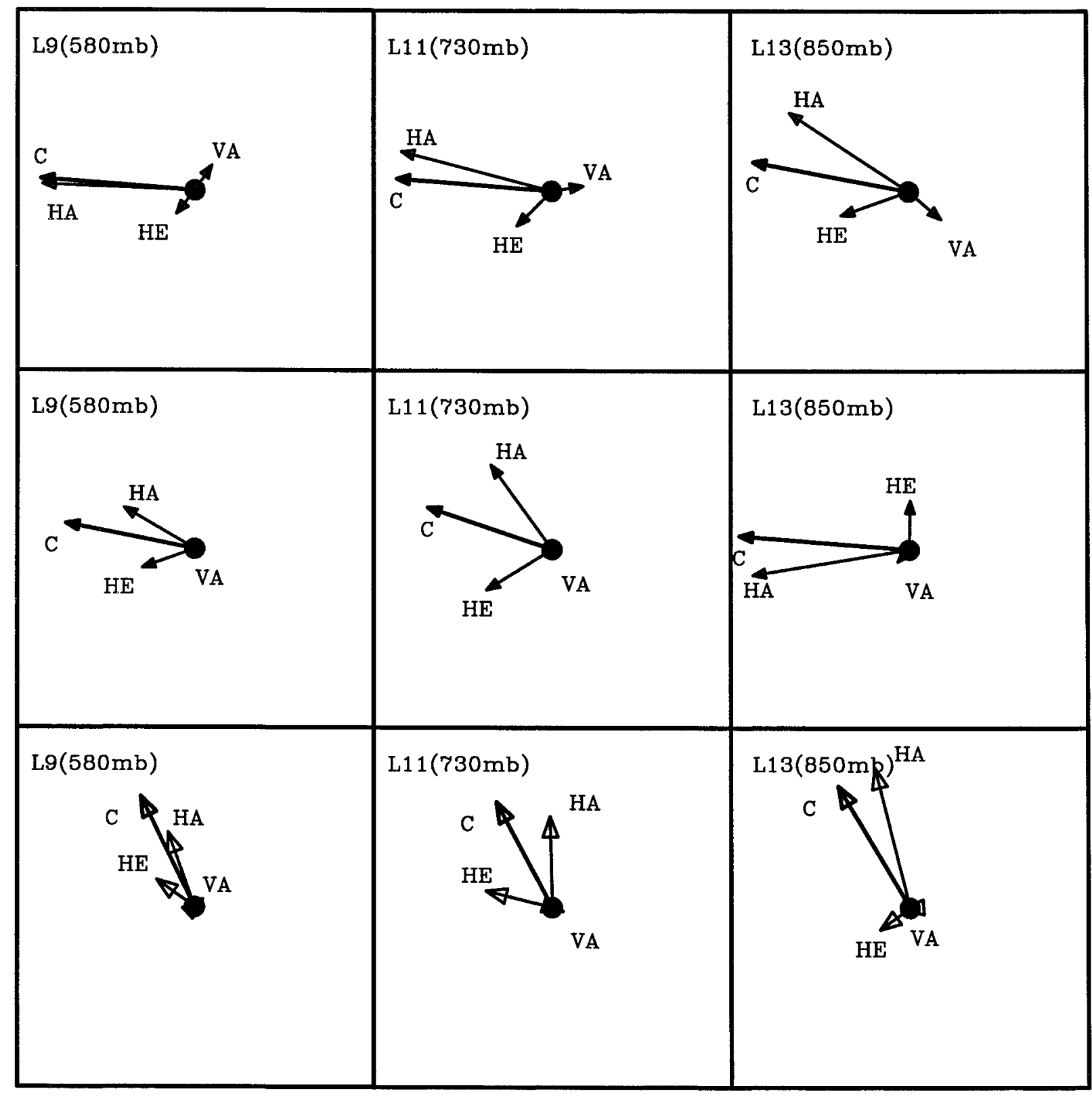

FIG. 3. Contributions of HA, VA, HE, and the total vortex motion speed (C) at levels $9(580 \mathrm{hPa}), 11(730 \mathrm{hPa})$, and 13 (850 hPa) at 48 $\mathrm{h}$ in (top) E1, (middle) E2, and (bottom) E3. The total motion speed (C) at level 13 is $4.7 \mathrm{~m} \mathrm{~s}^{-1}$ in E1, $3.4 \mathrm{~m} \mathrm{~s}^{-1}$ in E2, and $5.0 \mathrm{~m} \mathrm{~s}^{-1}$ in E3.

line) and PV tendency (dotted line). Due to the different vertical extent of the positive PV anomalies (Fig. 2), the mean speed in E1 is calculated from 440 to 850 $\mathrm{hPa}$, whereas it is calculated from 365 to $950 \mathrm{hPa}$ in E2 and E3. For comparison, the vertical mean speed of the asymmetric flow over the vortex core region $\left(\mathbf{V}_{m 1}\right)$ and the direct contribution of diabatic heating are also presented. As shown in Fig. 5, the mean speed estimated from the wavenumber-one component of the PV tendency $\left(\mathbf{C}_{e}\right)$ is nearly identical to the mean TC speed calculated from the center positions $(\mathbf{C})$. In contrast, the vertical mean speed of the asymmetric flow $\left(\mathbf{V}_{m 1}\right)$ differs from the mean TC speed. The direct contribution of diabatic heating can primarily account for the difference between $\mathbf{V}_{m 1}$ and $\mathbf{C}_{e}$. In other words, the diabatic heating contributes to the TC motion considerably.

The asymmetric flow in E1 does not arise only from the initial environmental flow $\left(-4 \mathrm{~m} \mathrm{~s}^{-1}\right)$. As shown in Fig. 1, the TC motion in the presence of diabatic heating exhibits a northward motion component. This deviation reaches its maximum around 36 h (Fig. 5). Except for the first $20 \mathrm{~h}$, this northward deflection pri- 
(a) E1

(b) E2 (c) E3

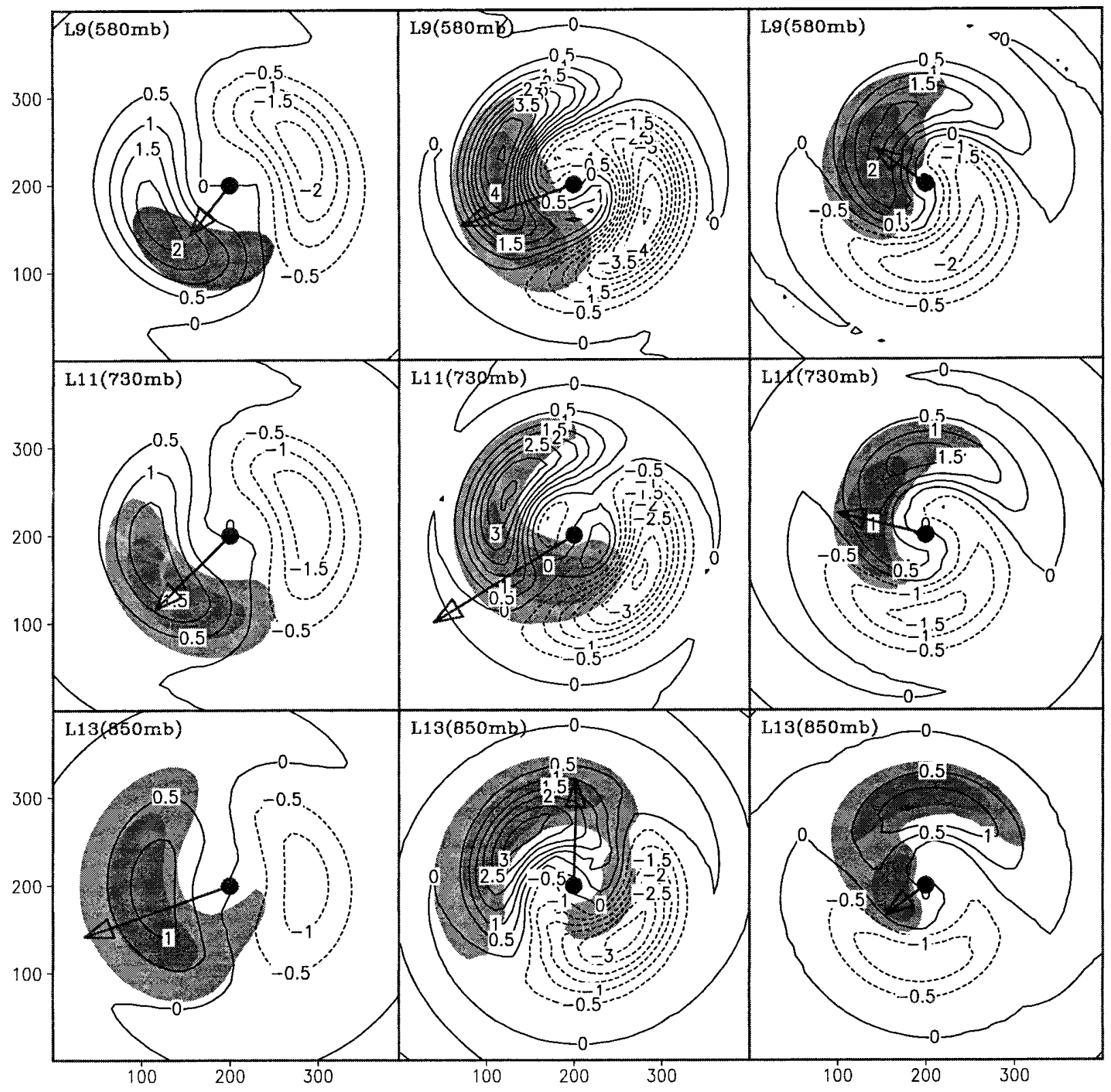

FIG. 4. The diabatic heating rate (contours) and vertical differential diabatic heating rate (shading) and the contribution of diabatic heating (arrows) to TC motion at $48 \mathrm{~h}$ in (a) E1, (b) E2, and (c) E3. The contour intervals are $0.5 \mathrm{~K} \mathrm{~h}^{-1}$, and the differential heating is calculated as the vertical difference of two adjacent levels.

marily arises from the steering of the asymmetric flow. An adiabatic experiment corresponding to E1 was run without the surface drag. In this dry experiment, the TC moves almost precisely with the easterly environmental flow of $4 \mathrm{~m} \mathrm{~s}^{-1}$. This suggests that the diabatic heating can also affect TC motion through the induced asymmetric wind field.

In E2, the TC motion speed is significantly different from the mean speed of the asymmetric flow, both in the zonal and meridional directions. The zonal mean speed of the asymmetric flow remains $2.5-3.0 \mathrm{~m} \mathrm{~s}^{-1}$ during the 72-h integration, while the zonal motion speed shows significant fluctuations with time. It reaches about $3.5 \mathrm{~m} \mathrm{~s}^{-1}$ at $48 \mathrm{~h}$. As suggested by Fig. 5, the resulting northward drift arises from the direct influence of diabatic heating.

In E3, the beta drift follows the asymmetric flow during the first several hours. Then the direct influence of diabatic heating plays an important role in the TC motion. Previous studies show that a TC in the adiabatic 

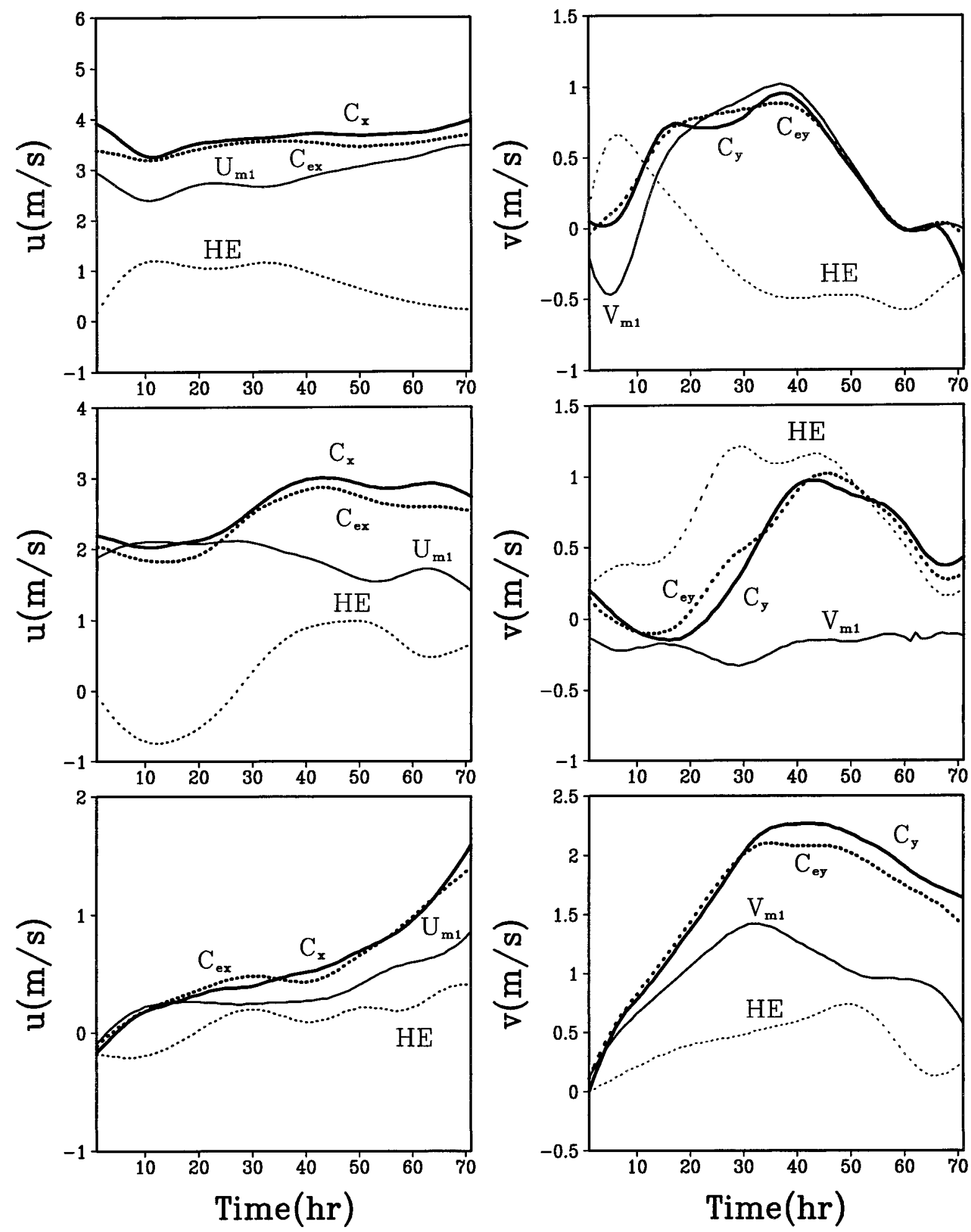

FIG. 5. Vertical mean zonal and meridional TC motion speed components calculated from the center positions $(\mathbf{C})$ and the PV tendency

$\left(\mathbf{C}_{e}\right)$, in comparison with the vertical mean asymmetrical flow speed $\left(\mathbf{V}_{m 1}\right)$ in (top) E1, (middle) E2, and (bottom) E3. 
experiment corresponding to E3 takes a track south of that in E3 (Wang and Holland 1996b). Figure 5 suggests that the direct influence of diabatic heating contributes to a northward track deviation. In addition, the direct influence of diabatic heating also makes the westward TC motion faster than that implied by the steering of the asymmetric flow. In summary, the diabatic TC motion is primarily determined by both the steering by the asymmetric flow and the direct influence of the vertical gradient of asymmetric diabatic heating.

\section{Existence of a steering level}

In the last section, we demonstrated that the vortex motion was primarily determined by the advection of the symmetric PV component by the asymmetric flow and the influence directly associated with the vertical gradient of asymmetric diabatic heating. Therefore, Eq. (1) can be rewritten as

$$
\left(\frac{\partial P}{\partial t}\right)_{1}=-\mathbf{V}_{1} \cdot \nabla P_{s}-\frac{g}{p_{s}}\left(f_{0}+\zeta_{s}\right) \frac{\partial}{\partial \sigma}\left(\frac{Q_{1}}{C_{p} \pi}\right),
$$

where $\left(f_{0}+\zeta_{s}\right)$ is the symmetric vertical component of the absolute vorticity, $P_{s}$ the symmetric component of PV, $Q_{1}$ the wavenumber-one component of diabatic heating, and $\pi$ is the Exner function. Since a TC that is dominated by the symmetric circulation tends to move to the region with maximum azimuthal wavenumberone component of the PV tendency (Wu and Wang 2000), we have

$$
\left(\frac{\partial P}{\partial t}\right)_{1}=-\mathbf{C} \cdot \nabla P_{s}
$$

Combining (2) and (3) yields

$$
\left(\mathbf{V}_{1}-\mathbf{C}\right) \cdot \nabla P_{s}=-\frac{g}{p_{s}}\left(f_{0}+\zeta_{s}\right) \frac{\partial}{\partial \sigma}\left(\frac{Q_{1}}{C_{p} \pi}\right),
$$

where $\left(\mathbf{V}_{1}-\mathbf{C}\right)$ is the relative flow, the asymmetric flow observed in the coordinates moving with a TC (Bender 1997). Equation (4) indicates that the vortex motion $(\mathbf{C})$ has the same speed and direction as the areal mean asymmetric flow $\left(\mathbf{V}_{1}\right)$ if the areal mean contribution of diabatic heating to the vortex motion vanishes at that level; that is, a steering level exists at which $\mathbf{V}_{1}$ = $\mathbf{C}$ (or asymmetric flow determines TC motion).

To search for the steering level in our diabatic experiments, the time mean vortex contributions of horizontal PV advection and diabatic heating are calculated in comparison with the time mean motion speed during the last $24 \mathrm{~h}$ of the integration (Fig. 6). Although the mean motion speed does not change much with height, the contributions of horizontal PV advection and diabatic heating vary significantly from level to level. As shown in Fig. 6, we can find a steering level in each experiment. For instance, the steering level is at level $10(650 \mathrm{hPa})$ in $\mathrm{E} 1$, at level $13(850 \mathrm{hPa})$ in $\mathrm{E} 2$, and between levels 13 and $14(850-900 \mathrm{hPa})$ in E3. Due to the well-coupled vertical structure of diabatic TCs (the mean vortex motion speed does not change much with height), this level can also be called the steering level of diabatic TCs.

In the previous studies, the appropriate level or layer mean to be used to indicate the steering current has been debated widely. It was shown that the $700-$ or $500-\mathrm{hPa}$ level provides the closest approximation (George and Gray 1976; Neumann 1979; Pike 1985). As suggested in Eq. (4), this steering level is primarily determined by the vertical profile of the asymmetric diabatic heating, which is modified by the Exner function. According to these three diabatic experiments, the steering level is located in the lower troposphere (650-900 hPa).

Many early studies of TC motion were involved in searching for the large-scale steering flow (see the reviews by Elsberry 1995 and Wang et al. 1998). A basic problem is how to define the large-scale steering flow. George and Gray (1976), Chan and Gray (1982), and Holland (1984) suggested using the basic current horizontally averaged over a $5^{\circ}-7^{\circ}$ latitude radial band surrounding the cyclone. Equation (4) indicates that the contribution of horizontal PV advection by the asymmetric flow $\left(-\mathbf{V}_{1} \cdot \nabla P_{s}\right)$ is determined by the horizontal PV gradient that is primarily confined within a radius of $200 \mathrm{~km}$ (Fig. 2). This suggests that the asymmetric flow within this region is most effective in steering a TC. This agrees with the results of the observational studies by Marks et al. (1992), Roux and Marks (1991), and Franklin et al. (1996), who show that the TC motion is best correlated with the layer mean flow averaged over the inner core region within $3^{\circ}$ latitude from the TC center.

\section{Vertical coupling}

In spite of the influence of the differential beta drift (E3) or the environmental shear (E2), the vertical coherent structure of TCs in these diabatic experiments is as well maintained as in E1 (Fig. 2). This agrees with the conclusions of previous studies (e.g., Wang and Holland 1996b) that diabatic heating can enhance the vertical coupling, so that a TC can resist the distortion caused by the vertical shear of relative asymmetric flows. An important question is how the diabatic heating can enhance the vertical coupling of the baroclinic TC circulation.

Figure 6 offers clues to the roles of horizontal PV advection and diabatic heating in the vertical coupling. The contributions of these two processes to the vortex motion show significant variations in the vertical. In E1, for instance, the contribution of the horizontal PV advection to the zonal movement is $4.1 \mathrm{~m} \mathrm{~s}^{-1}$ at level 11 and $1.2 \mathrm{~m} \mathrm{~s}^{-1}$ at level 15 (Fig. 6). Its meridional contribution ranges from $2.0 \mathrm{~m} \mathrm{~s}^{-1}$ at level 13 to $-0.6 \mathrm{~m}$ $\mathrm{s}^{-1}$ at level 15. Meanwhile, the zonal contribution of diabatic heating varies from $0.8 \mathrm{~m} \mathrm{~s}^{-1}$ at level 11 to 4.5 

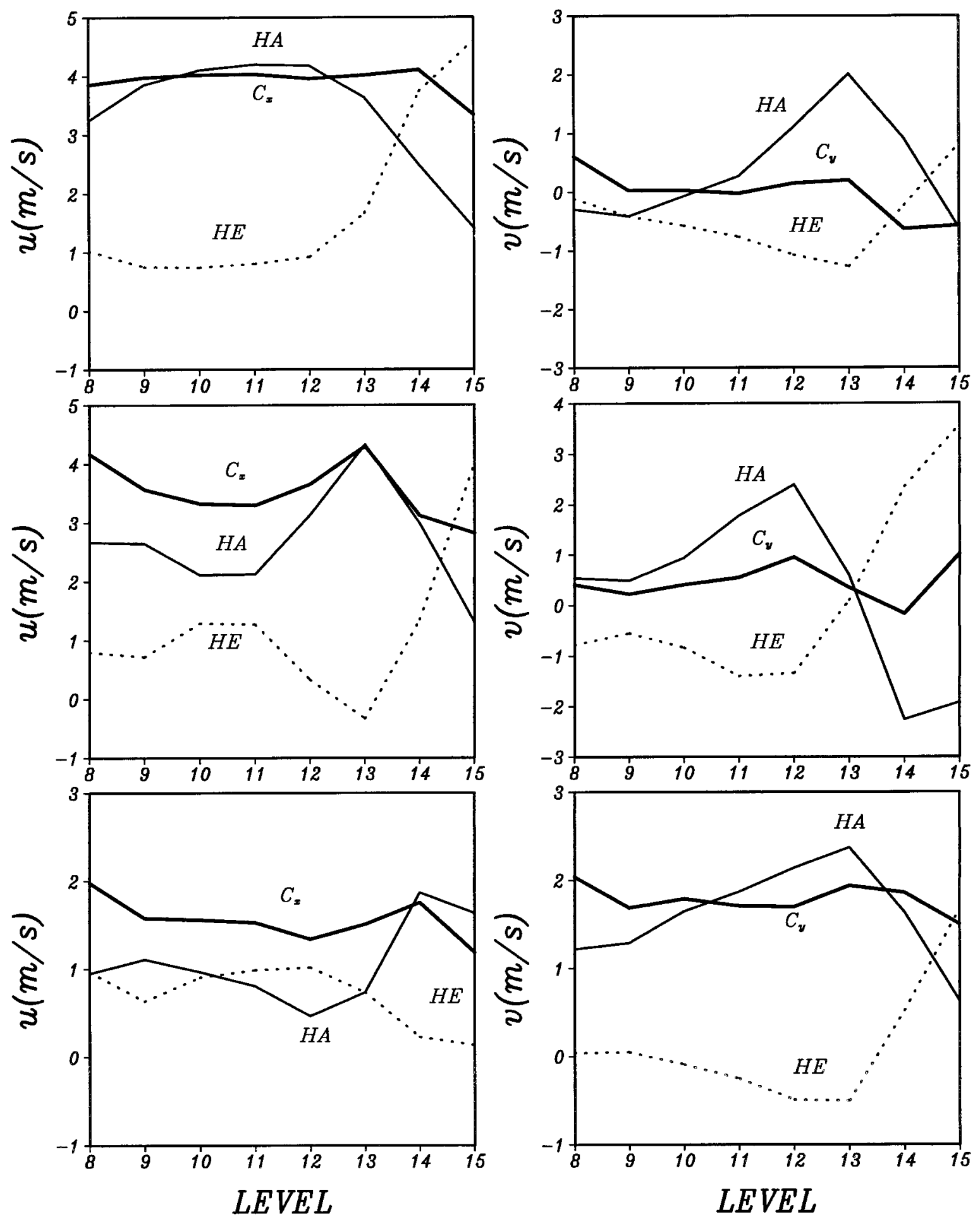

FIG. 6. Time mean (averaged over the last 24-h integration) contributions of HA and HE to the (left) zonal and (right) meridional of the TC motion vector (C) in (top) E1, (middle) E2, and (bottom) E3, respectively.

$\mathrm{m} \mathrm{s}^{-1}$ at level 15. Its meridional contribution, on the other hand, is $-1.0 \mathrm{~m} \mathrm{~s}^{-1}$ at level 13 and $0.8 \mathrm{~m} \mathrm{~s}^{-1}$ at level 15. The vertical variations of these two contributions are out of phase in the zonal and meridional directions. As a result, the vortices at different levels can still move at a similar speed. This out-of-phase relationship between asymmetric heating and winds is also clear in E2 and E3. This relationship manifests that the 

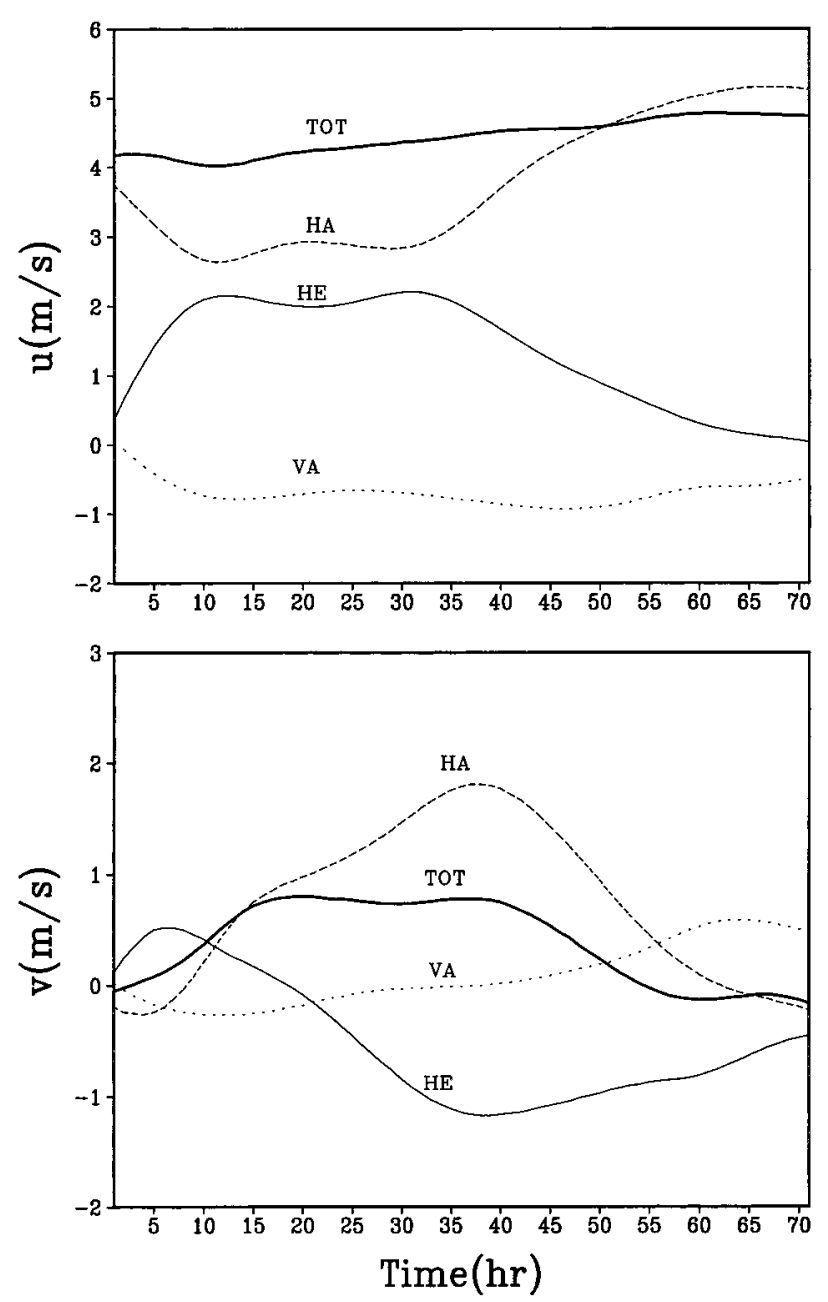

FIG. 7. (westward, top) Time series of the zonal and (northward, bottom) meridional contributions of HA, VA, and HE, and their sum (TOT) at level 11 in E1.

asymmetric relative flow and diabatic heating are closely associated with each other. Namely, the entrance region of wavenumber-one asymmetric flow is related to maximum diabatic heating or maximum vertical gradient of diabatic heating at the middle and lower levels. This agrees with Peng et al. (1999) that the entrance region of the wavenumber-one asymmetric flow in each level is related to the maximum of moisture convergence on that level.

However, such an out-of-phase relationship is not sufficient for the vertical coupling. In order to cancel the vertical tilt caused by the relative asymmetric flow or asymmetric diabatic heating, a fast adjustment between these two processes is necessary compared to the variations of TC speed. Figure 7 shows the time series of the contributions of these two physical processes to the motion. The immediate adjustment between these processes is clearly shown in Fig. 7. This fast adjustment is associated with the local response between the asymmetric flow and heating fields due to the decrease of the Rossby deformation radius (Ooyama 1982; Schubert and Hack 1982; Shapiro and Willoughby 1982; Hack and Schubert 1986). Therefore, diabatic heating enhances the vertical coupling of the circulations at different levels by such a fast adjustment.

\section{Summary}

In this paper, the influence of diabatic heating on TC motion and the physical mechanism for the heatingenhanced vertical coupling have been investigated through three diabatic numerical experiments, in which the TCs are affected by different environmental forcing. Our new findings are the following.

- The TC motion is not only determined from the steering by the asymmetric flow, but is also directly influenced by the asymmetric component of the diabatic heating.

- The asymmetric diabatic heating tends to move vortices to the region with maximum downward gradient of asymmetric diabatic heating.

- A level at which the influence of asymmetric diabatic heating vanishes can be found and the vortex at this level moves with the areal mean asymmetric flow. This level can be called the steering level of a diabatic TC due to the well-coupled vertical structure of diabatic TCs. Such a level cannot be found in the absence of heating (Wu and Wang 2001).

- At the steering level, the advection by the asymmetric flow is weighted by the horizontal gradient of $P_{s}$, which is primarily confined within a radius of 200 $\mathrm{km}$. Therefore, the asymmetric flow in the core region can more effectively affect the TC motion. This agrees with results obtained by observational studies (Marks et al. 1992; Roux and Marks 1991; Franklin et al. 1996).

- The heating-enhanced vertical coupling is realized through a fast adjustment between the relative asymmetric flow and the asymmetric diabatic heating. Due to this adjustment, the vertical shear in the relative asymmetric flow and the vertical profile of the asymmetric diabatic heating are always in a state such that their contributions to the vertical tilt tend to offset each other.

It has been demonstrated that diabatic heating is an important physical process affecting TC motion and is also closely associated with the development of the wind fields in the TC core region. Further studies should be focused on how and why the asymmetric convection develops and interacts with the vortex and environmental wind fields.

Acknowledgments. We would like to thank two anonymous reviewers for their careful review and valuable comments. We also thank Dr. Y. Wang for his original code of the hurricane model, and Mr. C. Orndorff for his comments. This research is supported by the Marine 
Meteorology Program of the Office of Naval Research.

\section{REFERENCES}

Bender, M. A., 1997: The effect of relative flow on the asymmetric structure in the interior of hurricanes. J. Atmos. Sci., 54, 703724.

Chan, J. C.-L., and W. M. Gray, 1982: Tropical cyclone motion and surrounding flow relationship. Mon. Wea. Rev., 110, 1354-1374

Dengler, K., and M. J. Reeder, 1997: The effects of convection and baroclinicity on the motion of tropical-cyclone-like vortices. Quart. J. Roy. Meteor. Soc., 123, 699-725.

Elsberry, R. L., 1988: Tropical cyclone motion. A Global View of Tropical Cyclones, R. L. Elsberry, Ed., Marine Meteorology Program, U.S. Office of Naval Research, $185 \mathrm{pp}$.

Fiorino, M. J., and R. L. Elsberry, 1989: Some aspects of vortex structure related to tropical cyclone motion. J. Atmos. Sci., 46, 975-990.

Flatau, M., W. H. Schubert, and D. E. Stevens, 1994: The role of baroclinic processes in tropical cyclone motion: The influence of vertical tilt. J. Atmos. Sci., 51, 2589-2601.

Frank, W. M., and E. A. Ritchie, 1999: Effects of environmental flow upon tropical cyclone structure. Mon. Wea. Rev., 127, 2044 2061.

Franklin, J. L., S. E. Feuer, J. Kaplan, and S. D. Aberson, 1996: Tropical cyclone motion and surrounding flow relationship: Searching for beta gyres in omega dropwindsonde datasets. Mon Wea. Rev., 124, 64-84.

George, J. E., and W. M. Gray, 1976: Tropical cyclone motion and surrounding parameter relationships. J. Appl. Meteor., 15, 1252 1264.

Hack, J. J., and W. H. Schubert, 1986: Nonlinear response of atmospheric vortices to heating by organized cumulus convection. J. Atmos. Sci., 43, 1559-1573.

Holland, G. J., 1984: Tropical cyclone motion: A comparison of theory and observation. J. Atmos. Sci., 41, 68-75.

— clone tracks. J. Atmos. Sci., 50, 1254-1266.

Jones, S. C., 1995: The evolution of vortices in vertical shear. Part 1: Initially barotropic vortices. Quart. J. Roy. Meteor. Soc., 121, $821-851$.

Kuo, H. L., 1974: Further studies of the parameterization of the influence of cumulus convection on large scale flow. J. Atmos. Sci., 31, 1232-1240.

Marks, F. D., Jr., R. A. Houze Jr., and J. F. Gamache, 1992: Dualaircraft investigation of the inner core of Hurricane Norbert. Part I: Kinematic structure. J. Atmos. Sci., 49, 919-942.

Neumann, C. J., 1979: A guide to Atlantic and Eastern Pacific models for the prediction of tropical cyclone motion. NOAA Tech. Memo. NWS NHC-11, 26 pp.

—, and J. G. Boyd, 1962: Hurricane movement and variable location of high energy spot in wall cloud radar echo. Mon. Wea. Rev., 90, 371-374.

Ooyama, K. V., 1982: Conceptual evolution of the theory and modeling of tropical cyclones. J. Meteor. Soc. Japan, 60, 369-379.

Peng, M. S., B. F. Jeng, and R. T. Williams, 1999: A numerical study on tropical cyclone intensification. Part I: Beta effect and mean flow effect. J. Atmos. Sci., 56, 1404-1423.

Pike, A. C., 1985: Geopotential heights and thicknesses as predictors of Atlantic tropical cyclone motion and intensity. Mon. Wea. Rev., 113, 931-939.

Raymond, D. J., 1992: Nonlinear balance and potential-vorticity thinking at large Rossby number. Quart. J. Roy. Meteor. Soc., 118, 987-1015.

Roux, F., and F. D. Marks Jr., 1991: Eyewall evolution in hurricane Hugo deduced from successive airborne Doppler observations. Preprints, 19th Conf. on Hurricanes and Tropical Meteorology, Miami, FL, Amer. Meteor. Soc., 558-563.

Schubert, W. H., and J. J. Hack, 1982: Inertial stability and tropical cyclone development. J. Atmos. Sci., 39, 1687-1697.

Shapiro, L. J., 1992: Hurricane vortex motion and evolution in a three-layer model. J. Atmos. Sci., 49, 140-153.

_ , and H. E. Willoughby, 1982: Response of balanced hurricanes to local sources of heat and momentum. J. Atmos. Sci., 39, 378394

_ , and J. L. Franklin, 1995: Potential vorticity in Hurricane Gloria. Mon. Wea. Rev. 123, 1465-1475.

Tuleya, R. E., and Y. Kurihara, 1984: The formation of comma vortices in a tropical numerical simulation model. Mon. Wea. Rev., 112, 491-502.

Wang, B., and X. Li, 1992: The beta drift of three-dimensional vortices: A numerical study. Mon. Wea. Rev., 120, 579-593.

— , R. L. Elsberry, Y. Wang, and L. Wu, 1998: Dynamics of tropical cyclone motion: A review. Sci. Atmos. Sinica, 12, 1-12.

Wang, Y., 1998: On the bogusing of tropical cyclone in numerical models: The influence of vertical structure. Meteor. Atmos. Phys., 65, 153-170.

— Part I: Adiabatic vortices. J. Atmos. Sci., 53, 411-427.

— and _ 1996b: The beta drift of baroclinic vortices. Part II: Diabatic vortices J. Atmos. Sci., 53, 3737-3756.

— vertical shear. J. Atmos. Sci., 53, 3313-3332.

Willoughby, H. E., 1988: Linear motion of a shallow-water barotropic vortex. J. Atmos. Sci., 45, 1906-1928.

_- 1992: Linear motion of a shallow water barotropic vortex as an initial-value problem. J. Atmos. Sci., 47, 242-264.

__ , F. D. Marks Jr., and R. J. Feinberg, 1984: Stationary and moving convective bands in hurricanes. J. Atmos. Sci., 41, 3189-3211.

Wu, C.-C., and K. A. Emanuel, 1993: Interaction of a baroclinic vortex with background shear: Application to hurricane movement. J. Atmos. Soc., 50, 62-76.

$\longrightarrow$, and — 1995a: Potential vorticity diagnostics of hurricane movement. Part I: A case study of Hurricane Bob (1991). Mon. Wea. Rev., 123, 69-92.

$\ldots$, and —_ 1995b: Potential vorticity diagnostics of hurricane movement. Part II: Tropical Storm Ana (1991) and Hurricane Andrew (1992). Mon. Wea. Rev., 123, 93-109.

— - and Y. Kurihara, 1996: A numerical study of the feedback mechanisms of hurricane-environment interaction on hurricane movement from the potential vorticity perspective. J. Atmos. Sci., 53, 2264-2282.

Wu, L., 1999: Study of tropical cyclone motion with a coupled hurricane-ocean model. Ph.D dissertation, Department of Meteorology, University of Hawaii, $212 \mathrm{pp}$.

—_ and B. Wang, 2000a: A potential vorticity tendency diagnostic approach for tropical cyclone motion. Mon. Wea. Rev., 128, 1899-1911.

—_, and —, 2001: Movement and vertical coupling of diabatic baroclinic tropical cyclones. J. Atmos. Sci., 58, 1801-1814. 